\title{
The Visceral Adiposity Index Is a Predictor of Incident Chronic Kidney Disease: A Population-Based Longitudinal Study
}

\author{
Ryo Bamba $^{a}$ Takuro Okamura ${ }^{a}$ Yoshitaka Hashimoto $^{a}$ \\ Masahide Hamaguchi ${ }^{a}$ Akihiro Oborab Takao Kojimab Michiaki Fukui ${ }^{b}$ \\ aDepartment of Endocrinology and Metabolism, Kyoto Prefectural University of Medicine, \\ Graduate School of Medical Science, Kyoto, Japan; ${ }^{b}$ Department of Gastroenterology, \\ Asahi University Hospital, Gifu, Japan
}

\section{Keywords}

Chronic kidney disease $\cdot$ Risk factors · Visceral adiposity index

\begin{abstract}
Background and Aims: Visceral adiposity index (VAI), calculated with body mass index, high density lipoprotein-cholesterol, triglycerides and waist circumference, has been proposed as a marker of visceral fat accumulation and dysfunction in adipose tissue. Methods: The impact of VAI on incident chronic kidney disease (CKD) in a historical cohort study of 15,159 $(8,260$ men and 6,899 women) participants was investigated. CKD was defined when estimated glomerular filtration rate was $<60 \mathrm{~mL} / \mathrm{min} / 1.73 \mathrm{~m}^{2}$ or proteinuria (positive: $\geq 1+$ ). We divided the participants into 2 groups according to sex and into quartiles according to VAI (Q1-4). We performed Cox proportional hazard models, adjusting for age, smoking status, exercise, alcohol consumption, systolic blood pressure, hemoglobin A1c, uric acid, and creatinine. $\boldsymbol{R e}$ sults: During the median 3.3-year follow-up for men and 3.2-year follow-up for women, 1,078 participants (629 men and 449 women) developed CKD. The 4,000 days cumulative incidence rate of CKD for men and women were 3.7 and 3.9\% in Q1, 5.2 and 5.9\% in Q2, 6.5 and $7.0 \%$ in $\mathrm{Q} 3$, and 8.4 and 9.3\% in Q4 respectively. Compared to Q1, the hazard ratios of incident CKD in Q2, Q3 and Q4 for men and women were $1.23(95 \% \mathrm{Cl} 0.91-1.66, p=0.184)$ and $1.30(0.87-$ $1.96, p=0.203), 1.42(1.06-1.90, p=0.018)$ and $1.38(0.94-2.05, p=0.105)$, and $1.51(1.12-2.02$, $p=0.006)$ and $1.65(1.12-2.46, p=0.013)$ respectively. Additionally, the area under the curve of VAl for incidence of CKD was superior to that of VAl in men $(0.595 \mathrm{vs.} 0.552, p<0.001)$ and equal to in women $(0.597$ vs. $0.591, p=0.708)$. Conclusions: The VAI can be a predictor of incident CKD.




\section{Kidney \\ Blood Pressure \\ Research}

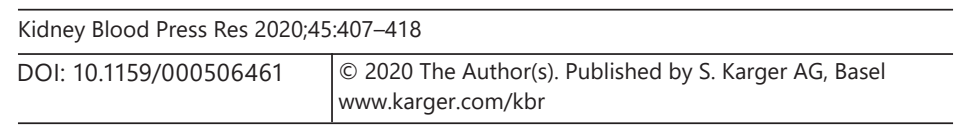

Bamba et al.: The VAI Is a Predictor of Incident CKD: A Population-Based Longitudinal Study

\section{Introduction}

Chronic kidney disease (CKD) is a public health problem, which represents a significant global health burden [1]. CKD remains asymptomatic until the stage of disease progresses. Moreover, CKD has been demonstrated to increase the risk of cardiovascular disease [2] and mortality [3]. Therefore, both prediction and prevention of CKD are important for not only prevention of end-stage of renal dysfunction but also decreased risk of cardiovascular disease and mortality.

Previous studies demonstrated that body mass index (BMI) is associated with glomerular filtration rate (GFR) [4]. Furthermore, several studies reported that the excessive visceral fat accumulation causes insulin resistance [3] and is the risk of metabolic complications such as impairment of glucose and lipid metabolism [5]. Visceral fat accumulation and insulin resistance play critical roles in the pathogenesis of CKD [5]. On the other hand, the visceral adiposity index (VAI) has been proposed as a reliable marker of visceral fat accumulation and dysfunction [6], and has been proved to be a strong association with type 2 diabetes [7] and cardiovascular events [8]. Similarly, it was reported that VAI is related with prevalence of CKD in a cross-sectional study [9]. In fact, while obesity predicts for CKD, the "reverse epidemiology" predicts lower mortality in obese subjects with CKD [10], which means that not only assessment of obesity may be important but also that of visceral fat obesity is important. Therefore, we performed this retrospective cohort study to investigate the impact of VAI on incident CKD.

\section{Materials and Methods}

\section{Study Design and Study Participants}

The NAfld in Gifu Area, Longitudinal Analysis cohort study is an ongoing prospective cohort study that began in 1994 [11]. For this population-based longitudinal analysis, we extracted the participants from a medical examination program at Asahi University Hospital (Gifu, Japan). This medical examination program is aimed to detect chronic diseases and their risk factors and promote public health. More than 8,000 participants annually registered and $60 \%$ of them receive 1-2 exams per year [12]. In this study, we investigated the impact of VAI on incident CKD, using the NAfld in Gifu Area, Longitudinal Analysis database. We extracted the participants who received the medical examination program from 1994 to 2016. We excluded the participants with medication at the baseline examination (Fig. 1). Approval for the study was obtained from the research Ethics Committees of the Asahi University Hospital, and written informed consent for their data to be used was obtained from all participants.

\section{Standardized Questionnaire for Lifestyle Factors}

In order to determine the lifestyle factors of participants, a standardized questionnaire was given to all participants [12]. We divided the participants into nonsmokers, ex-smokers and current smokers. Next, we asked about the type and amount of alcohol consumption per week during the prior month, then estimating the mean ethanol intake per week. Lastly, we defined the participants who performed any kind of sport regularly at least once a week as regular exercisers [13].

\section{Data Collection}

BMI was defined as weight in kilograms divided by height in meters squared. The participants' levels of several factors including fasting plasma glucose, triglycerides (TG), high-density lipoprotein (HDL) cholesterol and creatinine were measured using venous 


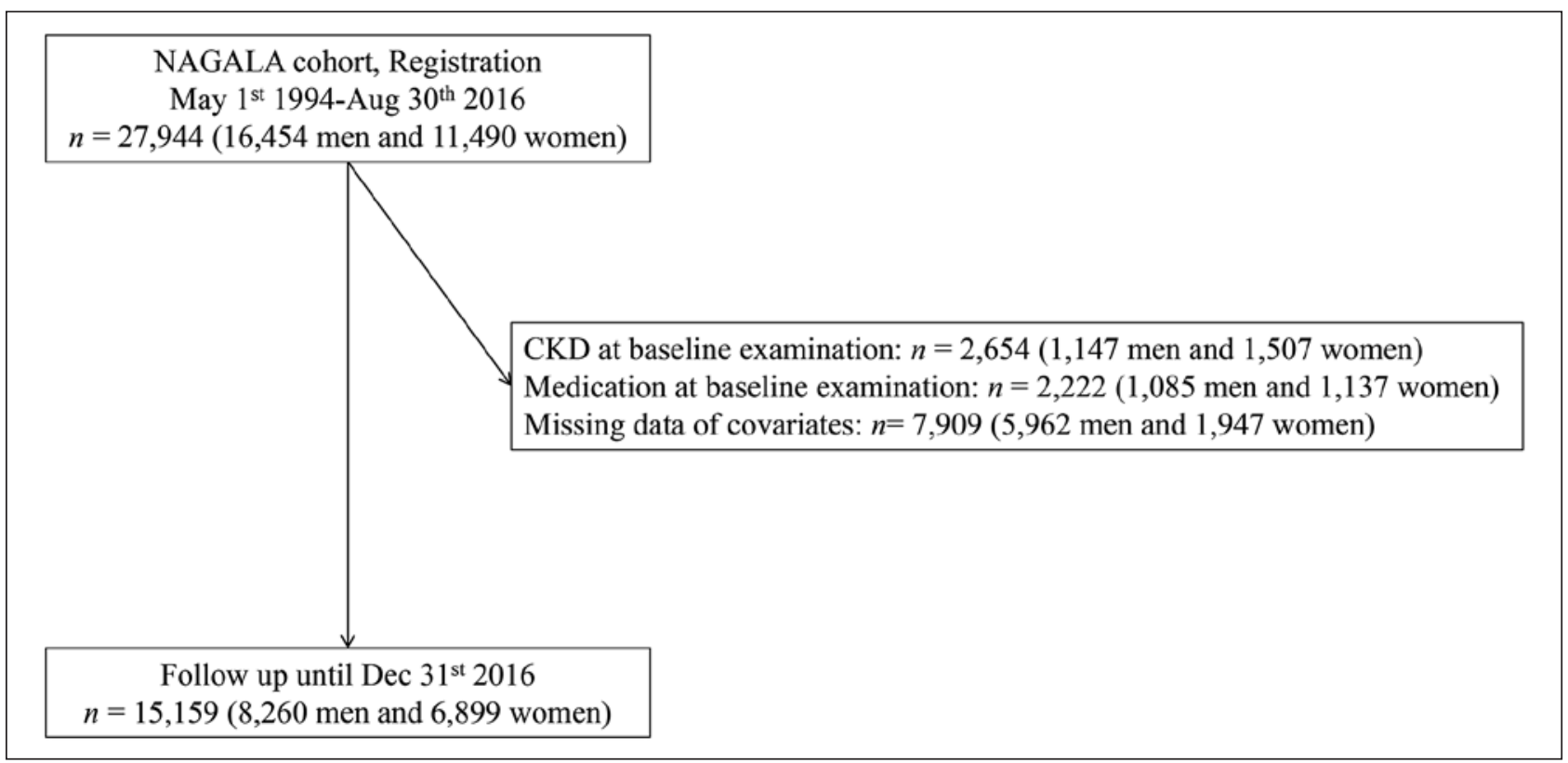

Fig. 1. Participant registration. NAGALA, NAfld in Gifu Area, Longitudinal Analysis; CKD, chronic kidney disease.

blood after an overnight fast. We used the Japanese Society of Nephrology equation for calculating each patient's estimated GFR (eGFR): eGFR $\left(\mathrm{mL} / \mathrm{min} / 1.73 \mathrm{~m}^{2}\right)=194 \times$ serum creatinine ${ }^{-1.094} \times \operatorname{age}^{-0.287}(\times 0.739$ for women $)$ [14]. Spot morning urine sample was measured.

\section{VAI Formulas}

We calculated VAI using the formulas [6]. Men: VAI = (waist circumference [WC] $/ 39.68$ $+[1.88 \times \mathrm{BMI}]) \times(\mathrm{TG} / 1.03) \times(1.31 / \mathrm{HDL}) ;$ women: $\mathrm{VAI}=(\mathrm{WC} / 36.58+[1.89 \times \mathrm{BMI}]) \times$ $(\mathrm{TG} / 0.81) \times(1.52 / \mathrm{HDL})$. Both TG and HDL levels are expressed in mmol/L. VAI is a novel genderspecific index based on $\mathrm{WC}, \mathrm{BMI}, \mathrm{TG}$, and $\mathrm{HDL}$, and a valuable indicator of visceral adipose function and insulin sensitivity, and its increase is strongly associated with cardiometabolic risk [6].

\section{Definition of CKD}

CKD was defined as decreased eGFR or presence of proteinuria. GFR was estimated using the Japanese Society of Nephrology equation. When eGFR was $<60 \mathrm{~mL} / \mathrm{min} / 1.73 \mathrm{~m}^{2}$, it was defined as having CKD. In addition, dipstick test in fasting morning urine was used for diagnosis of proteinuria (positive: $\geq 1+: \geq 30 \mathrm{mg} / \mathrm{dL}$ ) $[15,16]$.

\section{Statistical Analysis}

Statistical analyses were performed using JMP version 12.0 software (SAS Institute, Cary, NC, USA) and a $p$ value $<0.05$ was considered significant. Means or frequencies of potential confounding variables were calculated, and continuous variables are presented as the mean (SD). Because alcohol consumption was non-normal distribution, we performed logarithmic transformation before using as covariates. We divided the participants into men and women because the distribution of VAI differed between sexes. We evaluated the $p$ values using one-way analysis of variance for continuous variables and chi-square test for categorical variables, respectively. Moreover, we categorized the participants into 4 groups according to 
Table 1. Characteristics of study participants at baseline examination

\begin{tabular}{lccc}
\hline & Men & Women & $p$ value \\
\hline Number & 8,260 & 6,899 & $<0.001$ \\
Age, years & $41.9(9.0)$ & $41.5(9.2)$ & $<0.001$ \\
BMI, kg/m & $23.2(3.2)$ & $20.9(3.0)$ & $<0.001$ \\
SBP, mm Hg & $119.9(14.0)$ & $109.8(14.1)$ & $<0.001$ \\
Diastolic blood pressure, mm Hg & $75.2(10.1)$ & $67.7(9.8)$ & $<0.001$ \\
Fasting plasma glucose, mg/dL & $98.6(15.6)$ & $90.6(9.2)$ & $<0.001$ \\
Hemoglobin A1c, \% & $5.28(0.58)$ & $5.2(0.4)$ & $<0.001$ \\
TG, mmol/L & $1.3(0.9)$ & $0.6(0.4)$ & $<0.001$ \\
HDL cholesterol, mmol/L & $1.3(0.36)$ & $1.9(0.5)$ & $<0.001$ \\
Creatinine, mmol/dL & $78.1(9.1)$ & $56.9(7.5)$ & $<0.001$ \\
\hline eGFR, mL/min/1.73 m ${ }^{2}$ & $77.9(11.4)$ & $81.7(13.3)$ & $<0.001$ \\
AST, IU/L & $21.0(10.6)$ & $17.1(9.3)$ & $<0.001$ \\
ALT, IU/L & $25.4(16.5)$ & $15.0(12.9)$ & $<0.001$ \\
Gamma-glutamyltransferase, IU/L & $28.3(28.1)$ & $14.1(8.9)$ & - \\
Smoking status & - & - & $<0.001$ \\
Never smoker & $2,802(33.9)$ & $5,774(83.8)$ & $<0.001$ \\
Ex-smoker & $2,312(28.0)$ & $568(8.2)$ & $<0.001$ \\
Current smoker & $3,146(38.1)$ & $557(8.1)$ & $<0.001$ \\
Habit of exercise & $1,422(17.2)$ & $982(14.2)$ & $<0.001$ \\
Alcohol consumption, g/week & $93.5(151.6)$ & $22.3(71.0)$ & $<0.001$ \\
VAI & $1.2(1.6)$ & $0.76(0.8)$ & \\
\hline
\end{tabular}

BMI, body mass index; TG, triglycerides; HDL, high-density lipoprotein; eGFR, estimated glomerular filtration rate; AST, aspartate aminotransferase; ALT, alanine aminotransferase; VAI, visceral adiposity index; SBP, systolic blood pressure.

quartile of VAI: $<0.51$ in men and $<0.37$ in women (quartile 1 [Q1]), $\geq 0.51$ and $<0.86$ in men and $\geq 0.37$ and $<0.56$ in women (quartile 2 [Q2]), $\geq 0.86$ and $\leq 1.49$ in men and $\geq 0.56$ and $\leq 0.89$ in women (quartile 3 [Q3]) and $>1.49$ in men and $>0.89$ in women (quartile [Q4]). Categorical variables were compared among the groups by Pearson's qui-square test, and continuous variables were compared by one-way analysis of variance and Tukey honestly significant difference test respectively. We used Kaplan-Meier analysis for a graphical presentation of time to incident CKD, and log-rank test to evaluate difference among the groups according to VAI. We performed a Bonferroni correction and considered that a $p$ value $<0.0083$ was statistically significant in the log-rank test.

Cox proportional hazard model was used to calculate unadjusted and adjusted hazard ratio (HR) and 95\% CI for incident CKD. We adjusted for age, smoking status, exercise, alcohol consumption, systolic blood pressure (SBP), hemoglobin A1c, uric acid, and creatinine. Additionally, the area under the curves (AUCs) of VAI and BMI for the incidence of CKD was calculated by the receiver operating characteristic curve, and scatterplot analyses were performed to investigate the association between eGFR and VAI.

\section{Results}

The baseline characteristics of the participants are summarized in Tables 1, 2. VAI in men was significantly higher than that in women (1.23 [1.59] vs. 0.76 [0.77], $p<0.001$ ). BMI, SBP, diastolic blood pressure, fasting plasma glucose, TG, uric acid, aspartate aminotransferase 


\section{Research}

Bamba et al.: The VAl Is a Predictor of Incident CKD: A Population-Based Longitudinal
Study

Table 2. Clinical characteristics of the participants in the groups based on the quartiles of the VAI

\begin{tabular}{|c|c|c|c|c|c|}
\hline VAI & $\begin{array}{l}\text { Q1 } \\
(0.048 \leq \text { VAI, } \\
\text { VAI }<0.51)\end{array}$ & $\begin{array}{l}\text { Q2 } \\
(0.51 \leq \mathrm{VAI}, \\
\mathrm{VAI}<0.86)\end{array}$ & $\begin{array}{l}\text { Q3 } \\
(0.86 \leq \mathrm{VAI}, \\
\mathrm{VAI}<1.49)\end{array}$ & $\begin{array}{l}\text { Q4 } \\
(1.49 \leq \mathrm{VAI}, \\
\mathrm{VAI}<51.17)\end{array}$ & $p$ value \\
\hline \multicolumn{6}{|l|}{ Gender, men } \\
\hline Number & 2,065 & 2,065 & 2,065 & 2,065 & \\
\hline Age, years & $40.4(0.2)$ & $41.7(0.2)^{\dagger}$ & $42.7(0.2)^{\dagger, \ddagger}$ & $42.6(0.2)^{\dagger, \ddagger, \S}$ & $<0.001$ \\
\hline $\mathrm{BW}, \mathrm{kg}$ & $71.4(0.3)$ & $66.2(0.3)^{\dagger}$ & $62.7(0.3)^{\dagger, \ddagger}$ & $57.5(0.3)^{\dagger, \ddagger, \S}$ & $<0.001$ \\
\hline $\mathrm{BMI}, \mathrm{kg} / \mathrm{m}^{2}$ & $23.7(0.1)$ & $22.5(0.1)^{\dagger}$ & $21.7(0.1)^{\dagger}$ & $20.5(0.1)^{\dagger, \ddagger, \S}$ & $<0.001$ \\
\hline $\mathrm{SBP}, \mathrm{mm} \mathrm{Hg}$ & $115.6(0.3)$ & $118.4(0.3)^{\dagger}$ & $121.0(0.3)^{\dagger}$ & $124.7(0.3)^{\dagger, \ddagger, \S}$ & $<0.001$ \\
\hline Diastolic blood pressure, $\mathrm{mm} \mathrm{Hg}$ & $71.6(0.2)$ & $74.1(0.2)^{\dagger}$ & $76.1(0.2)^{\dagger}$ & $78.9(0.2)^{\dagger, \ddagger, \S}$ & $<0.001$ \\
\hline Fasting plasma glucose, $\mathrm{mmol} / \mathrm{L}$ & $5.2(0.01)$ & $5.2(0.01)$ & $5.3(0.01)$ & $5.4(0.01)^{\dagger, \ddagger, \S}$ & $<0.001$ \\
\hline Hemoglobin A1c, \% & $5.2(0.1)$ & $5.2(0.1)$ & $5.2(0.1)^{\ddagger}, \S$ & $5.2(0.1)^{t, \ddagger, \S}$ & 0.229 \\
\hline $\mathrm{TG}, \mathrm{mmol} / \mathrm{L}$ & $0.5(0.02)$ & $0.8(0.02)^{\dagger}$ & $1.1(0.02)^{\dagger, \ddagger}$ & $2.2(0.02)^{\dagger, \ddagger, \S}$ & $<0.001$ \\
\hline HDL cholesterol, $\mathrm{mmol} / \mathrm{L}$ & $1.7(0.06)$ & $1.4(0.06)^{\dagger}$ & $1.2(0.06)^{\dagger, \ddagger}$ & $1.0(0.06)^{\dagger, \ddagger, \S}$ & $<0.001$ \\
\hline Creatinine, $\mathrm{mmol} / \mathrm{dL}$ & $76.6(0.2)$ & $77.9(0.2)^{\dagger}$ & $78.4(0.2)^{\dagger, \ddagger}$ & $79.5(0.2)^{\dagger, \ddagger, \S}$ & $<0.001$ \\
\hline $\mathrm{eGFR}, \mathrm{mL} / \mathrm{min} / 1.73 \mathrm{~m}^{2}$ & $80.6(0.2)$ & $78.1(0.2)^{\dagger}$ & $77.1(0.2)^{\dagger, \ddagger}$ & $75.8(0.2)^{\dagger, \ddagger, \S}$ & $<0.001$ \\
\hline AST, IU/L & $19.4(0.2)$ & $19.4(0.2)^{\dagger}$ & $21.3(0.2)^{\dagger, \ddagger}$ & $23.8(0.2)^{\dagger, \ddagger, \S}$ & $<0.001$ \\
\hline ALT, IU/L & $19.5(0.3)$ & $21.8(0.3)^{\dagger}$ & $27.0(0.3)^{\dagger, \neq}$ & $33.1(0.3)^{t, \neq, \S}$ & $<0.001$ \\
\hline Gamma-glutamyltransferase, IU/L & $21.1(0.6)$ & $23.9(0.6)$ & $29.5(0.6)$ & $38.8(0.6)^{\dagger, \ddagger}$ & $<0.001$ \\
\hline Smoking status & & & & & 0.019 \\
\hline Never smoker & $862(30.8)$ & $718(25.6)$ & 657 (31.8) & $565(27.3)$ & \\
\hline Ex-smoker & $579(25.0)$ & $600(26.0)$ & $536(25.8)$ & $536(23.2)$ & \\
\hline Current smoker & $617(19.6)$ & 747 (23.7) & $813(25.8)$ & $969(30.8)$ & \\
\hline Habit of exercise & $270(17.0)$ & $325(20.4)$ & $313(19.6)$ & $308(19.4)$ & 0.082 \\
\hline Alcohol consumption, g/week & $94.9(3.3)$ & $88.8(3.3)$ & $89.6(3.3)$ & $100.6(3.4)$ & 0.046 \\
\hline Creatinine/BW $(\times 100)$ & $\mathrm{Q} 1(<1.17)$ & Q2 $(\geq 1.17,<1.33)$ & Q3 $(\geq 1.33,<1.51)$ & Q4 ( $\geq 1.51)$ & $p$ value \\
\hline \multicolumn{6}{|l|}{ Gender, women } \\
\hline Number & 1,730 & 1,715 & 1,722 & 1,732 & \\
\hline Age, years & $38.9(0.2)$ & $40.2(0.2)$ & $41.8(0.2)$ & $45.0(0.2)^{\dagger, \ddagger}$ & $<0.001$ \\
\hline $\mathrm{BW}, \mathrm{kg}$ & $49.6(0.2)$ & $51.2(0.2)^{\dagger}$ & $52.5(0.2)^{\dagger, \ddagger}$ & $56.7(0.2)^{\dagger, \ddagger, \S}$ & $<0.001$ \\
\hline BMI, $\mathrm{kg} / \mathrm{m}^{2}$ & $20.0(0.1)$ & $20.2(0.1)^{\dagger}$ & $20.9(0.1)^{\dagger, \ddagger}$ & $22.8(0.1)^{\dagger, \ddagger, \S}$ & $<0.001$ \\
\hline $\mathrm{SBP}, \mathrm{mm} \mathrm{Hg}$ & $106.3(0.3)$ & $107.5(0.3)^{\dagger}$ & $109.4(0.3)^{t, \ddagger}$ & $116.0(0.3)^{\dagger, \ddagger, \S}$ & $<0.001$ \\
\hline Diastolic blood pressure, $\mathrm{mm} \mathrm{Hg}$ & $65.4(0.2)$ & $66.0(0.2)^{\dagger}$ & $67.5(0.2)^{\dagger, \ddagger}$ & $71.8(0.2)^{\dagger, \ddagger}$ & $<0.001$ \\
\hline Fasting plasma glucose, $\mathrm{mmol} / \mathrm{L}$ & $5.0(0.01)$ & $5.0(0.01)^{\dagger}$ & $5.1(0.01)^{\dagger}$ & $5.3(0.01)^{\dagger, \ddagger}$ & $<0.001$ \\
\hline Hemoglobin A1c, \% & $5.2(0.01)$ & $5.2(0.01)^{\dagger}$ & $5.1(0.01)^{\dagger, \ddagger}$ & $5.3(0.01)^{\dagger, \ddagger, \S}$ & $<0.001$ \\
\hline $\mathrm{TG}, \mathrm{mmol} / \mathrm{L}$ & $0.2(0.01)$ & $0.4(0.01)^{\dagger}$ & $0.6(0.01)^{\dagger, \ddagger}$ & $1.0(0.01)^{\dagger, \ddagger, \S}$ & $<0.001$ \\
\hline HDL cholesterol, mmol/L & $2.3(0.01)$ & $2.0(0.01)^{\dagger}$ & $1.9(0.01)^{\dagger}$ & $1.6(0.01)^{\dagger}$ & $<0.001$ \\
\hline Creatinine, $\mathrm{mmol} / \mathrm{dL}$ & $56.3(0.2)$ & $56.9(0.2)^{\dagger}$ & $57.1(0.2)^{\dagger, \ddagger}$ & $57.2(0.2)^{\dagger, \ddagger}$ & 0.0009 \\
\hline $\mathrm{eGFR}, \mathrm{mL} / \mathrm{min} / 1.73 \mathrm{~m}^{2}$ & $84.2(0.3)$ & $82.3(0.3)^{\dagger}$ & $81.1(0.3)^{\dagger, \ddagger}$ & $79.1(0.3)^{t, \neq, \S}$ & $<0.001$ \\
\hline $\mathrm{AST}, \mathrm{IU} / \mathrm{L}$ & $16.8(0.2)$ & $16.4(0.2)$ & $16.9(0.2)$ & $18.2(0.2)$ & $<0.001$ \\
\hline ALT, IU/L & $14.0(0.3)$ & $13.9(0.3)^{\dagger}$ & $14.6(0.3)^{\dagger, \ddagger}$ & $17.4(0.3)^{\dagger, \ddagger, \S}$ & $<0.001$ \\
\hline Gamma-glutamyltransferase, IU/L & $12.8(0.2)$ & $13.0(0.2)^{\dagger}$ & $14.0(0.2)^{\dagger, \ddagger}$ & $16.7(0.2)^{\dagger, \ddagger, \S}$ & $<0.001$ \\
\hline Smoking status & & & & & $<0.001$ \\
\hline Never smoker & $1,462(25.3)$ & $1,465(25.4)$ & $1,458(24.1)$ & $1,389(24.1)$ & \\
\hline Ex-smoker & $167(29.4)$ & $142(25.0)$ & $117(20.6)$ & $142(25.0)$ & \\
\hline Current smoker & $101(18.1)$ & $108(19.4)$ & $147(26.4)$ & $201(36.1)$ & \\
\hline Habit of exercise & $261(26.6)$ & $222(22.6)$ & $245(25.0)$ & $254(25.9)$ & 0.30 \\
\hline Alcohol consumption, g/week & $22.1(1.7)$ & $24.5(1.7)$ & $21.6(1.7)$ & $20.8(1.7)^{\dagger, \ddagger}$ & 0.45 \\
\hline
\end{tabular}

Data are expressed as mean (SD) or number (\%) of subjects.

$P$ values by one-way analysis of variance for continuous variables and chi-square test for categorical variables. The analyses of continuous among four groups were performed by Tukey HSD test.

${ }^{\dagger} p<0.05$ versus Q1.

${ }^{\ddagger} p<0.05$ versus Q2.

$\S p<0.05$ versus Q3.

VAI, visceral adiposity index; BMI, body mass index; BW, body weight; HDL, high-density lipoprotein; TG, triglycerides; Q, quartile; eGFR, estimated glomerular filtration rate; AST, aspartate aminotransferase; ALT, alanine aminotransferase; SBP, systolic blood pressure. 


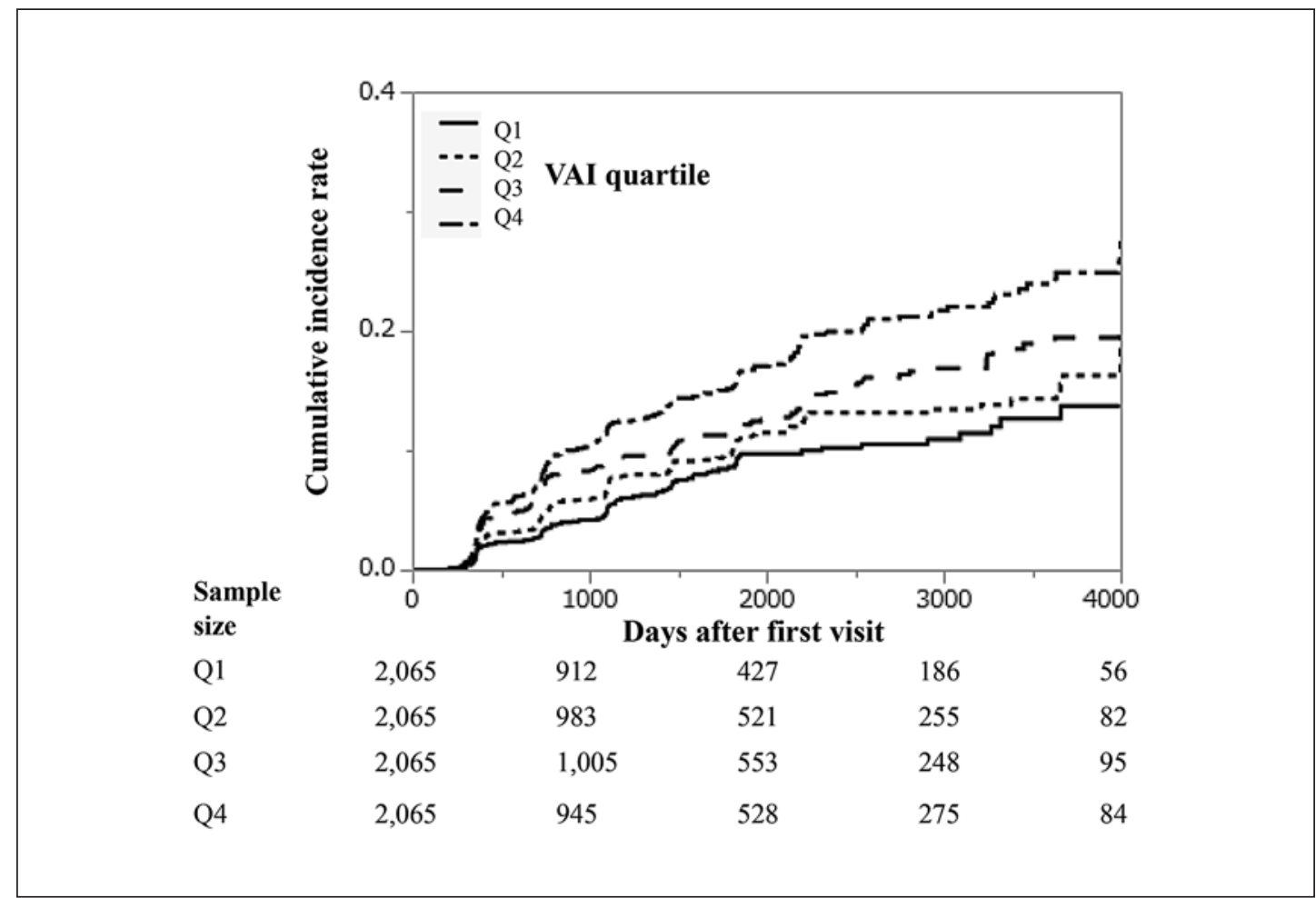

Fig. 2. Kaplan-Meier analysis of incident CKD in 8,260 men. Log-rank tests were used to determine the association among the VAI quartiles. Bonferroni correction was performed to correct familiar error, and a $p$ value $<0.0083$ was considered significant. Log-rank test results, compared to Q1, Q2: $p=0.074, \mathrm{Q} 3: p<0.001$, $\mathrm{Q} 4: p<0.001$. Log-rank test compared to Q2, Q3: $p=0.311, \mathrm{Q} 4: p<0.001$. Log-rank test compared to Q3, Q4: $p=0.005$. VAI, visceral adiposity index.

and alanine aminotransferase of Q4 were the highest and HDL cholesterol and eGFR of Q4 were the lowest, followed in order by Q3, Q2, and Q1 in men (Table 2). Similarly, Age, body weight, BMI, SBP, and TG of Q4 were the highest and eGFR of Q4 were the lowest, followed in order by Q3, Q2, and Q1 in women.

During the median 3.3-year follow-up for men and 3.2-year follow-up for women, 1,078 participants (629 men and 449 women) developed CKD. The 4,000 days cumulative incidence rate of CKD were 3.7\% for men and 3.9\% for women in Q1, 5.2\% for men and 5.9\% for women in Q2, 6.5\% for men and 7.0\% for women in Q3 and 8.4\% for men and 9.3\% for women in Q4. Compared to Q1, Q4 showed a significantly higher risk of incident CKD in both men $(p<0.001$; Fig. 2) and women ( $p<0.001$; Fig. 3).

Compared to Q1, the HRs of incident CKD in Q2, Q3, and Q4 were 1.23 (95\% CI 0.91-1.66, $p=0.184)$ in men and $1.30(0.87-1.96, p=0.203)$ in women, $1.42(1.06-1.90, p=0.018)$ in men and $1.38(0.94-2.05, p=0.105)$ in women and $1.51(1.12-2.02, p=0.006)$ in men and $1.65(1.12-2.46, p=0.013)$ in women, respectively (Table 3$)$. In addition, the HRs of VAI were $1.08(1.03-1.14, p=0.003)$ in men and $1.18(1.01-1.39, p=0.034)$. According to the receiver operating characteristic analysis, the AUC of VAI in men was significantly higher than that of BMI ( 0.595 vs. $0.552, p<0.001)$. Furthermore, in women, that of AUC was almost equal to that of BMI ( 0.597 vs. $0.591, p=0.708)$. In scatterplot analyses, VAI was negatively associated with eGFR in both men and women (men: $\beta=-0.066, p<0.001$; women: $\beta=-0.12$, $p<0.001$; Fig. 4). 
Kidney

Blood Pressure

Research

\begin{tabular}{l|l}
\hline Kidney Blood Press Res 2020;45:407-418 \\
\hline DOI: 10.1159/000506461 & $\begin{array}{l}\text { @ 2020 The Author(s). Published by S. Karger AG, Basel } \\
\text { www.karger.com/kbr }\end{array}$ \\
\hline
\end{tabular}

Bamba et al.: The VAI Is a Predictor of Incident CKD: A Population-Based Longitudinal Study

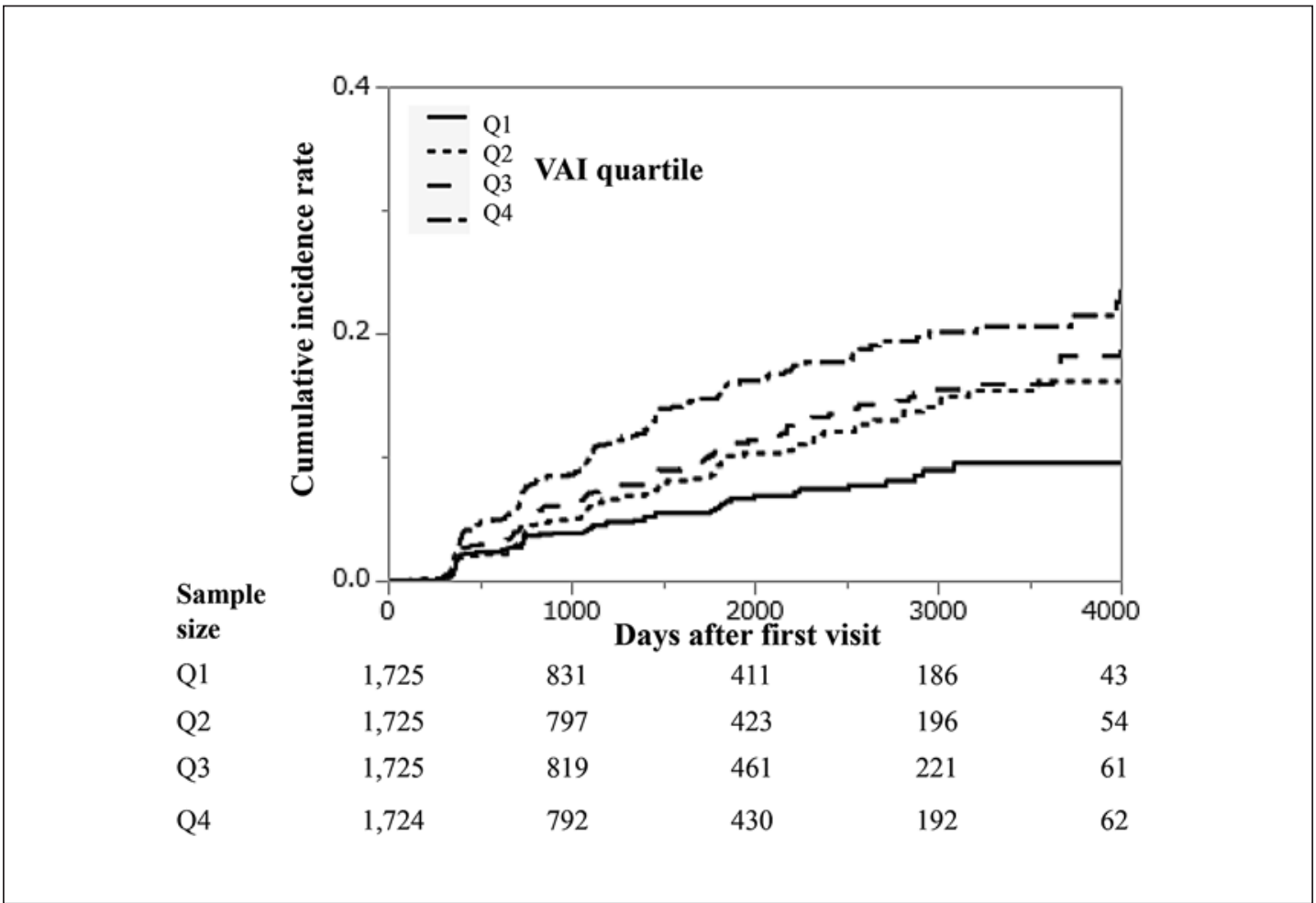

Fig. 3. Kaplan-Meier analysis of incident CKD in 6,899 women. Log-rank tests were used to determine the association among the VAI quartiles. Bonferroni correction was performed to correct familiar error, and a $p$ value $<0.0083$ was considered significant. Log-rank test results compared to Q1, Q2: $p=0.006, \mathrm{Q} 3: p<0.001$, $\mathrm{Q} 4: p<0.001$. Log-rank test compared to Q2, Q3: $p=0.016, \mathrm{Q} 4: p<0.001$. Log-rank test compared to Q3, Q4: $p=0.006$. VAI, visceral adiposity index.

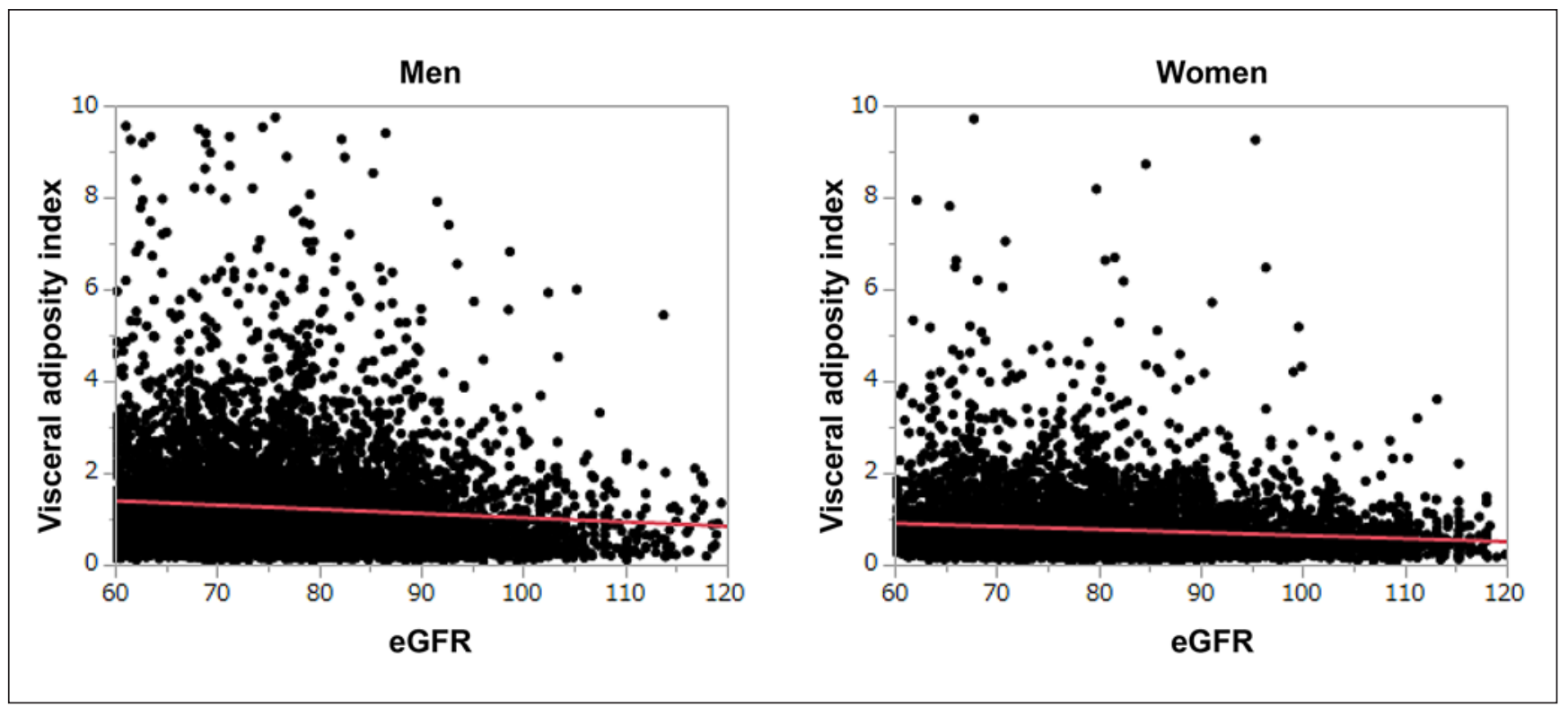

Fig. 4. Scatterplot analyses of the association between eGFR and VAI. VAI was negatively associated with eGFR in both men and women (men: $\beta=-0.066, p<0.001$; women: $\beta=-0.12, p<0.001$ ). VAI, visceral adiposity index; eGFR, estimated glomerular filtration rate. 
Kidney

Blood Pressure

Research
Kidney Blood Press Res 2020;45:407-418

DOI: $10.1159 / 000506461$

(c) 2020 The Author(s). Published by S. Karger AG, Basel www.karger.com/kbr

Bamba et al.: The VAI Is a Predictor of Incident CKD: A Population-Based Longitudinal Study

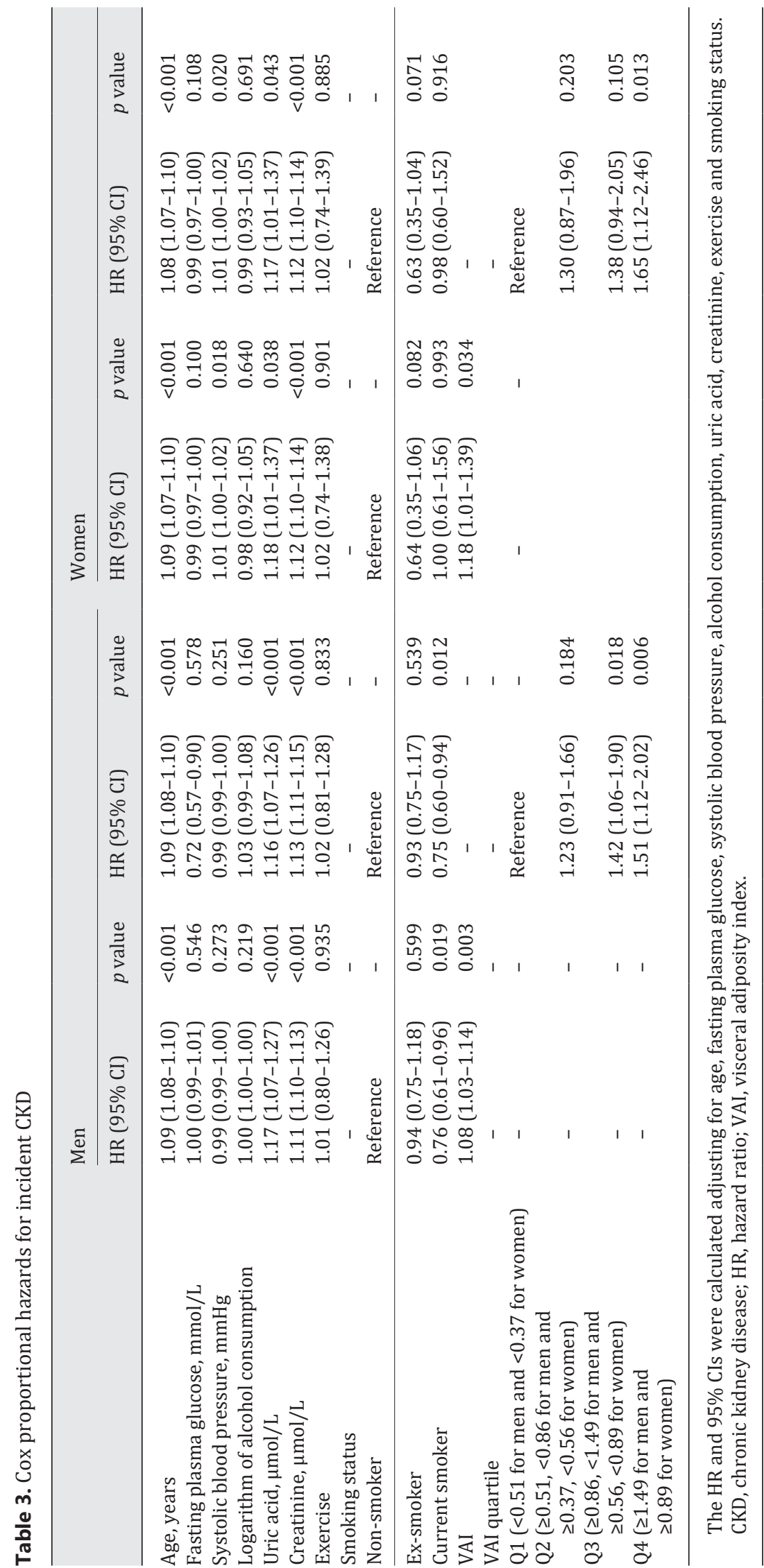




\section{Discussion}

In this cohort study of over 15,000 Japanese individuals, for the first time, our study revealed that higher VAI was associated with incident CKD both in men and women. CKD, which has become a public health problem [1], is the risk of cardiovascular disease [2] and increased mortality [3]. Hence, intervention at an early stage of the disease is desirable. Chen et al. [9] reported the association between higher VAI and CKD in cross-sectional analysis. However, there is no report of the association between VAI and incident CKD. The VAI, calculated with BMI, HDL cholesterol, TG and WC, was originally proposed as a diagnostic tool for cardiovascular and cerebrovascular event [6], and several following studies demonstrated the association between high VAI score and incident cardiovascular disease [8], type 2 diabetes, [7] and hypertension [16]. On the other hand, WC, which is a useful marker of visceral fat accumulation, did not show significant risk both in men and women (men: HR $1.01,95 \%$ CI 0.99-1.02, $p=0.133$; women: $1.01,0.99-1.03, p=0.078$ ), and VAI could be a useful marker for incident CKD. Moreover, many studies showed a link between BMI and GFR [4]. The AUC of VAI was significantly greater than that of BMI in men and equal to in women. The HR of VAI for incident CKD defined as proteinuria was 1.09 in men (95\% CI 1.04-1.14, $p<0.001)$ and 0.94 in women $(0.65-1.36, p=0.740)$. Additionally, that resulted as decline in eGFR $\left(<60 \mathrm{~mL} / \mathrm{min} / 1.73 \mathrm{~m}^{2}\right)$ was 1.09 in men $(1.06-1.12, p<0.001)$ and 1.33 in women (1.21-1.44, $p<0.001)$. VAI did not show a significant risk for incident CKD defined as proteinuria in women. Possible explanation is that incident CKD defined as proteinuria in women was only 38 in the observation period, and therefore, the statistical power of tests might not be enough.

VAI is associated with visceral fat accumulation and dysfunction [6]. Some potential explanations are proposed to support our findings. Visceral adipose tissue releases free fatty acid (FFA) by lipolysis, which induces the inflammatory responses in several cells, such as macrophages and adipocytes $[17,18]$. The mitochondria play a key role in the metabolism of FFA and determine the fate of lipotoxicity [19]. Renal lipotoxicity is known to cause detrimental effects on the kidney by several mechanisms of action including reclusion of proinflammatory factors [20], oxidative and endoplasmic reticulum (ER) stress development [21, 22], insulin resistance [23], or renin-angiotensin aldosterone system overactivation [24]. Adipokines, which were secreted from adipose tissue, are involved in kidney damage through the mediating endothelial dysfunction, inducing oxidative stress and inflammation as well as stimulating the renal sympathetic nervous activity $[19,24]$. Oxidative stress may contribute to the development of ER stress by promoting protein unfolding and activating the unfolded protein response. In fact, it has been shown that dietary saturated FFAs induce ER stress in kidneys of animal models [21] and in renal culture cells [25]. Moreover, insulin resistance affects the kidney, especially the podocytes. Podocytes are the most sensitive renal cells to insulin and thus the insulin resistance associated to obesity largely affects them [26]. Indeed, studies performed in cultured podocytes have shown that treatment with palmitic acid promotes insulin resistance and changes in the cytoskeleton leading to apoptosis [22]. Furthermore, the adipose tissue also secretes all components of the renin-angiotensin aldosterone system. There is an overactivation of this axis that may translate into hyperfiltration under the situation of obesity. Under this situation, the glomerulus manifests a big physical stress and consequential damages for the glomerular filtration barrier, especially the podocytes [23]. This might translate into a decreased GFR.

The strengths of our study include using the standardized questionnaire for lifestyle factors, and the relatively large population-based longitudinal research. Our study has also several limitations. First, we assessed proteinuria with urine dipstick test and did not quantify proteinuria. A dipstick test is often used in general practice and $<1+$ or less than trace has a 


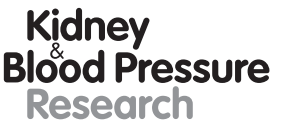

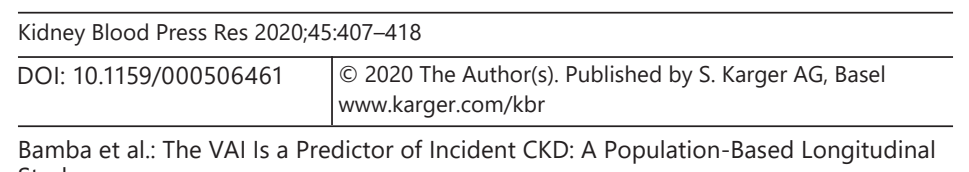

Bamba et al.: The VAI Is a Predictor of Incident CKD: A Population-Based Longitudinal
Study

high negative predictive value in the general community setting $[15,16]$. Additionally, we confirmed urine experiments once. If we performed urine experiments multiple times, we could evaluate CKD more accurately. Second, the follow-up was for a medium-term, and therefore, the statistical power might be limited. Third, sodium and protein intake are associated with pathogenesis of CKD [27, 28]. However, we did not have the data of them. If we had the data, we could more accurately investigate the association between VAI and incident CKD. Fourth, participants in this study received a health examination, and therefore, part of them might have changed and improved their lifestyles, which prevent incident CKD. Fifth, smoking is a well-known risk factor of incident CKD. However, non-smokers showed a significant higher risk compared to current smokers in men. In univariate analysis, current smoking did not show significant risk for incident CKD (HR 0.83, 95\% CI 0.69-1.01, $p=0.064$ ). Age, creatinine, and VAI, which were the strong factors for incident CKD, might lead to the result that non-smoker showed significant higher risk for incident CKD compared to current smoker. Sixth, because sever CKD, defined as eGFR $<30 \mathrm{~mL} / \mathrm{min} / 1.73 \mathrm{~m}^{2}$, is more important risk factor for mortality, the association between VAI and incident sever CKD is important. Unfortunately, however, the number of participants with severe CKD defined on eGFR $<30 \mathrm{~mL} /$ $\mathrm{min} / 1.73 \mathrm{~m}^{2}$ was 35 in men and 7 in women during observation period. Therefore, we could not perform accurate analysis. Additionally, we have excluded the participants with any medication at baseline. Therefore, participants with any immunological or other diseases contributing toward CKD at baseline should have been excluded. However, there is a possibility that participants who developed any diseases contributing toward CKD could not be excluded during observation period. Lastly, almost all participants were Japanese; therefore, it is uncertain whether our findings can be generalized to other ethnic groups.

In conclusion, we demonstrated, for the first time, that VAI can be a predictor of incident CKD.

\section{Acknowledgements}

We thank all of the staff members in the medical health checkup center at Asahi University Hospital.

\section{Statement of Ethics}

All procedures performed in studies involving human participants were in accordance with the ethical standards of the institutional research committee at which the studies were conducted and with the 1964 Helsinki Declaration and its later amendments or comparable ethical standards.

\section{Disclosure Statement}

Y.H. received grants from Asahi Kasei Pharma outside the submitted work. M.F. reports grants from AstraZeneca plc, grants from Astellas Pharma Inc., grants from Nippon Boehringer Ingelheim Co., Ltd., grants from Daiichi Sankyo Co., Ltd., grants from Eli Lilly Japan K.K., grants from Kyowa Hakko Kirin Company Ltd., grants from Kissei Pharmaceutical Co., Ltd., grants from MSD K.K., grants from Mitsubishi Tanabe Pharma Corporation, grants from Novo Nordisk Pharma Ltd., grants from Sanwa Kagaku Kenkyusho Co., Ltd., grants from Sanofi K.K., grants from Ono Pharmaceutical Co., Ltd., and grants from Takeda Pharmaceutical Co., Ltd., 


\section{Kidney \\ Blood Pressure \\ Research}

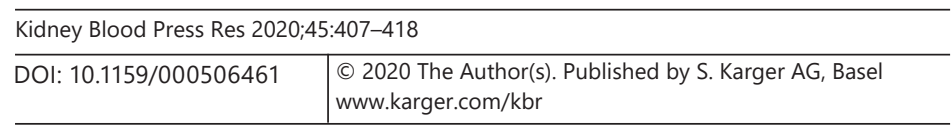

Bamba et al.: The VAI Is a Predictor of Incident CKD: A Population-Based Longitudinal Study

outside the submitted work. The sponsors were not involved in the study design; in the collection, analysis, interpretation of data; in the writing of this manuscript; or in the decision to submit the article for publication. The authors, their immediate families, and any research foundations with which they are affiliated have not received any financial payments or other benefits from any commercial entity related to the subject of this article. The authors declare that although they are affiliated with a department that is supported financially by pharmaceutical company, the authors received no current funding for this study and this does not alter their adherence to all the journal policies on sharing data and materials. The other authors have nothing to disclose.

\section{Funding Sources}

There are no funding sources to declare.

\section{Author Contributions}

R.B. and T.O. contributed to the data research and analyses and wrote the manuscript. Y.H. originated and designed the study, analyzed the data and reviewed the manuscript for intellectual content. M.H. contributed to the manuscript organization and reviewed and edited the manuscript. A.O. and T.K. originated the study, analyzed the data and contributed to the discussion. M.F. analyzed the data and reviewed and edited the manuscript. H.M. is the guarantor of this work and, as such, had full access to all of the data in the study and takes responsibility for the integrity of the data and the accuracy of the data analysis. All authors were involved in the writing of the manuscript and approved the manuscript's final version.

\section{Informed Consent}

Informed consent was obtained from all individual participants included in the study.

\section{References}

1 Levey AS, Atkins R, Coresh J, Cohen EP, Collins AJ, Eckardt KU, et al. Chronic kidney disease as a global public health problem: approaches and initiatives - a position statement from Kidney Disease Improving Global Outcomes. Kidney Int. 2007 Aug; 72(3):247-59.

2 Holman RR, Paul SK, Bethel MA, Neil HA, Matthews DR. Long-term follow-up after tight control of blood pressure in type 2 diabetes. N Engl J Med. 2008 Oct;359(15):1565-76.

3 Afkarian M, Sachs MC, Kestenbaum B, Hirsch IB, Tuttle KR, Himmelfarb J, et al. Kidney disease and increased mortality risk in type 2 diabetes. J Am Soc Nephrol. 2013 Feb;24(2):302-8.

4 Anastasio P, Viggiano D, Zacchia M, Altobelli C, Capasso G, Gaspare De Santo N. Delay in Renal Hemodynamic Response to a Meat Meal in Severe Obesity. Nephron. 2017;136(2):151-7.

5 Sanches FM, Avesani CM, Kamimura MA, Lemos MM, Axelsson J, Vasselai P, et al. Waist circumference and visceral fat in CKD: a cross-sectional study. Am J Kidney Dis. 2008 Jul;52(1):66-73.

6 Amato MC, Giordano C, Galia M, Criscimanna A, Vitabile S, Midiri M, et al.; AlkaMeSy Study Group. Visceral Adiposity Index: a reliable indicator of visceral fat function associated with cardiometabolic risk. Diabetes Care. 2010 Apr;33(4):920-2.

7 Zhang M, Zheng L, Li P, Zhu Y, Chang H, Wang X, et al. 4-Year Trajectory of Visceral Adiposity Index in the Development of Type 2 Diabetes: A Prospective Cohort Study. Ann Nutr Metab. 2016;69(2):142-9.

8 Kouli GM, Panagiotakos DB, Kyrou I, Georgousopoulou EN, Chrysohoou C, Tsigos C, et al. Visceral adiposity index and 10-year cardiovascular disease incidence: the ATTICA study. Nutr Metab Cardiovasc Dis. 2017 Oct; 27(10):881-9. 
9 Chen YC, Lai SH, Tsai YW, Chang SS. Visceral Adiposity Index as a Predictor of Chronic Kidney Disease in a Relatively Healthy Population in Taiwan. J Ren Nutr. 2018 Mar;28(2):91-100.

10 Kalantar-Zadeh K, Block G, Humphreys MH, Kopple JD. Reverse epidemiology of cardiovascular risk factors in maintenance dialysis patients. Kidney Int. 2003 Mar;63(3):793-808.

11 Hamaguchi M, Kojima T, Takeda N, Nakagawa T, Taniguchi H, Fujii K, et al. The metabolic syndrome as a predictor of nonalcoholic fatty liver disease. Ann Intern Med. 2005 Nov;143(10):722-8.

12 Okamura T, Hashimoto Y, Hamaguchi M, Obora A, Kojima T, Fukui M. Ectopic fat obesity presents the greatest risk for incident type 2 diabetes: a population-based longitudinal study. Int J Obes. 2019 Jan;43(1):139-48.

13 Ryu S, Chang Y, Kim DI, Kim WS, Suh BS. gamma-Glutamyltransferase as a predictor of chronic kidney disease in nonhypertensive and nondiabetic Korean men. Clin Chem. 2007 Jan;53(1):71-7.

14 Matsuo S, Imai E, Horio M, Yasuda Y, Tomita K, Nitta K, et al.; Collaborators developing the Japanese equation for estimated GFR. Revised equations for estimated GFR from serum creatinine in Japan. Am J Kidney Dis. 2009 Jun;53(6):982-92.

15 Hashimoto Y, Tanaka M, Okada H, Senmaru T, Hamaguchi M, Asano M, et al. Metabolically healthy obesity and risk of incident CKD. Clin J Am Soc Nephrol. 2015 Apr;10(4):578-83.

16 Yamamoto Y, Oi K, Chiba T, Shimazu S, Uchida T, Miyazawa Y. Basic studies of automatic urine analyzer "US-3100R" [in Japanese]. J Clin Lab Inst Reag. 2003;26:217-24.

17 Schilling JD, Machkovech HM, He L, Sidhu R, Fujiwara H, Weber K, et al. Palmitate and lipopolysaccharide trigger synergistic ceramide production in primary macrophages. J Biol Chem. 2013 Feb;288(5):2923-32.

18 Shi H, Kokoeva MV, Inouye K, Tzameli I, Yin H, Flier JS. TLR4 links innate immunity and fatty acid-induced insulin resistance. J Clin Invest. 2006 Nov;116(11):3015-25.

19 Zhu Y, Chen YL, Li C, Ding XY, Xu GY, Hu LL, et al. The effect of inhibition of endoplasmic reticulum stress on lipolysis in white adipose tissue in a rat model of chronic kidney disease. Acta Pharmacol Sin. 2014 Mar;35(3): 356-62.

20 Rüster C, Wolf G. Adipokines promote chronic kidney disease. Nephrol Dial Transplant. 2013 Nov;28(Suppl 4):iv8-14.

21 Welsh GI, Hale LJ, Eremina V, Jeansson M, Maezawa Y, Lennon R, et al. Insulin signaling to the glomerular podocyte is critical for normal kidney function. Cell Metab. 2010 Oct;12(4):329-40.

22 Li C, Lin Y, Luo R, Chen S, Wang F, Zheng P, et al. Intrarenal renin-angiotensin system mediates fatty acidinduced ER stress in the kidney. Am J Physiol Renal Physiol. 2016 Mar;310(5):F351-63.

23 Martínez-García C, Izquierdo-Lahuerta A, Vivas Y, Velasco I, Yeo TK, Chen S, et al. Renal Lipotoxicity-Associated Inflammation and Insulin Resistance Affects Actin Cytoskeleton Organization in Podocytes. PLoS One. 2015 Nov; 10(11):e0142291.

24 Kershaw EE, Flier JS. Adipose tissue as an endocrine organ. J Clin Endocrinol Metab. 2004 Jun;89(6):2548-56.

25 Zhu Q, Scherer PE. Immunologic and endocrine functions of adipose tissue: implications for kidney disease. Nat Rev Nephrol. 2018 Feb;14(2):105-20.

26 Sieber J, Lindenmeyer MT, Kampe K, Campbell KN, Cohen CD, Hopfer H, et al. Regulation of podocyte survival and endoplasmic reticulum stress by fatty acids. Am J Physiol Renal Physiol. 2010 Oct;299(4):F821-9.

27 Yoon CY, Noh J, Lee J, Kee YK, Seo C, Lee M, et al. High and low sodium intakes are associated with incident chronic kidney disease in patients with normal renal function and hypertension. Kidney Int. 2018 Apr;93(4): 921-31.

28 Haring B, Selvin E, Liang M, Coresh J, Grams ME, Petruski-Ivleva N, et al. Dietary Protein Sources and Risk for Incident Chronic Kidney Disease: Results From the Atherosclerosis Risk in Communities (ARIC) Study. J Ren Nutr. 2017 Jul;27(4):233-42. 